\title{
Verzwickte Verwandtschaftsverhältnisse: Kunstgeschichte, Visual Culture, Bildwissenschaft
}

Verwandtschaft ist bekanntlich eine Struktur, die ihre prekäre Balance der Kräfte wie ihre zeitliche Dauer sowohl in der Definition und Performanz der Rollen ihrer Akteure als auch in den Beziebungen zwischen ihnen, durch Abgrenzung wie durch Gabentausch, sicherzustellen versucht. Diachrone Dynamiken der Generationen wie synchrone der Konkurrenz um Anerkennung und Macht sind hier in einander verflochten. Bezogen auf die drei, Verwandten' im Titel könnte das heißen: Die Kunstgeschichte ist nun, mit der Jahrtausendwende, in den Status einer, Vater/Mutter-Disziplin hineingewachsen: traditionsbewußt, in den Augen der ,Söhne/Töchter 'Visual Culture Studies und Bildwissenschaft veraltet, engstirnig und nicht mehr auf dem letzten Stand. Ohne eines der Familienmitglieder bevorzugen zu wollen, möchte ich im folgenden kurz die Prozesse der Abgrenzung wie des Tausches, die im Namen des ,Bildes‘ und des ,Visuellen‘ als kleinsten gemeinsamen Nennern des Clans zu beobachten sind, nachvollziehen.

Um die Felder des Bildes und der Visualität finden seit Jahren wissenschaftspolitische Auseinandersetzungen der Zuständigkeiten statt. In den Termini ,Bild' und ,Visualität' zeichnen sich bereits die Unterschiede in den Strukturen und Kulturen von Wissen, Wissenschaften, Erkenntnishorizonten und Institutionen zwischen dem deutschsprachigen und angloamerikanischen Wissenschaftsbetrieb ab. Beteiligt sind im deutschsprachigen Raum für die Bildwissenschaft neben der Kunstgeschichte unter anderen die Literaturwissenschaft, die Psychoanalyse, die Soziologie, die Anthropologie, die Geschichte, die Kognitionswissenschaften, die Kommunikationswissenschaft, die Linguistik, die Neurowissenschaft, die Philosophie, diverse Naturwissenschaften. Gerade die Kunstgeschichte scheint recht isoliert dazustehen, während sich um das ,Bild“ Koalitionen zwischen bis dato eher ,bildfernen' Wissenschaften gebildet haben. ${ }^{1}$ In den USA formiert sich der Wissenschaftsverbund zur Visual Culture

1 Vgl. z. B. den Sammelband: Bildwissenschaft. Disziplinen, Themen, Methoden, hrsg. v. Klaus Sachs-Hombach, Frankfurt a. M. 2005. 
hingegen bisher neben der Kunstgeschichte aus Studies in den kulturorientierten Humanities wie der Kulturanthropologie, der Film- und Medienwissenschaft oder der Literaturwissenschaft. Auch hier jedoch ist die Kunstgeschichte keineswegs im Zentrum der wissenschaftspolitischen Formation Visual Culture. Ihre relative Isolation hat in den USA dazu geführt, daß viele KunstgeschichtlerInnen sich heute eher als Visual Culture-VertreterInnen denn als VertreterInnen ihrer Herkunftsdisziplin bezeichnen würden. Eine solche Strategie greift in Deutschland wohl deshalb nicht, weil es ein vergleichbar institutionalisiertes Feld (noch) nicht gibt, während die Universitäten in den USA seit zehn Jahren Studienprogramme zur Visual Culture etabliert haben, mit Beteiligten aus vielen Disziplinen. ${ }^{2}$ Die Annäherungen von KunsthistorikerInnen, vor allem in den Vereinigten Staaten, an die Visual Culture Studies gehen zumeist mit einem Leiden an ihrer Herkunftsdisziplin einher. ${ }^{3}$ Zwar mußte sich die US-amerikanische Kunstgeschichte nicht aus den nationalistischen Implikationen europäischer Kunstgeschichtsschreibung lösen, aber sie scheint belastet zu sein mit Verdikten des Formalismus, des Ästhetizismus (was oft genug als dasselbe erachtet wird), der Dominanz der weißen Oberschicht europäischer Provenienz.

Die Rhetorik von Auseinandersetzungen verläuft gerne über - negative Zuschreibungen, die der jeweils eigenen Profilierung dienen können, zum einen, wenn die ,junge' Generation entweder noch nicht weiß, ,wer' sie ist oder sein könnte, oder wenn sie aus dem Korsett einer nicht mehr zeitgemäßen Bestimmung und Praxis heraus will, zum anderen, wenn ,Vater/Mutter zur Rettung des Bestandes aufrufen. Für ersteres scheint die Kunstgeschichte als Negativfolie gute Dienste zu leisten: Der Bildwissenschaft ist ihr Gegenstandsbereich zu beschränkt, den Visual Culture Studies ist sie zu elitär, ästhetisch, unpolitisch, kolonial, national westlich hegemonial, bildungsbürgerlich und künstlergenialisch ausgerichtet - Einwände, die den KunsthistorikerInnen meiner Generation nur zu vertraut sind und die seit den siebziger Jahren in der Bundesrepublik zu verwandtschaftlich-generationalen Auseinandersetzungen innerbalb der Kunstgeschichte geführt haben, mit dem Resultat, daß sich die kunsthistorische Praxis enorm verändert hat.

2 Vgl. Margaret Dikovitskaya: Visual Culture. The Study of the Visual after the Cultural Turn, Cambridge 2005, mit einer institutionellen Geschichte des Feldes und ausführlichen Interviews einiger seiner ProtagonistInnen, darunter Michael Ann Holly, die zu Beginn der neunziger Jahre an der Rochester University das erste universitäre Studienprogramm mitgründete.

3 Vgl. z. B. Keith Moxey: The Social History of Art in the Age of Deconstruction, in: History of the Human Sciences 5/1, 1992, 37-46. Ein anderes Beispiel Douglas Crimp: Getting the Warhol we deserve, in: Social Text 59, 17/2, 1999, 49-65, ist eine Auseinandersetzung mit der traditionellen Kunstgeschichte aus der Perspektive einer Visual Culture im Sinne der Queer Studies. 
Soweit die drastisch verkürzte Bestandsaufnahme einer Situation, die sich entwickelte, nachdem die Ausrufung des pictorial turn ${ }^{4}$, dem in Deutschland der iconic turn ${ }^{5}$ folgte, Anlaß zu der Hoffnung gegeben hatte, den in der deutschen Kunstgeschichte ungeliebten linguistic turn und seine poststrukturalistischen französischen Verwandten ad acta legen und gleichzeitig die wissenschaftspolitische Bedeutung des Fachs stärken zu können. Inzwischen jedoch scheint es eine Art bösen Erwachens zu geben: Rund um das Bild wuchsen (die botanische Metapher scheint gar nicht so fehl am Platze zu sein) interdisziplinäre Wissenschaftscluster quer zu den Human-, Natur- und Technikwissenschaften, denen es gelungen ist, vom forschungspolitischen Hunger auf Interdisziplinarität ebenso wie von der Ausrufung des neuen Paradigmas zu profitieren. Die Kunstgeschichte jedoch ist in diesen Clustern schwach vertreten.

Das Erstaunen mancher KunsthistorikerInnen ist in Deutschland groß: Wie konnte das passieren, daß ausgerechnet jene Disziplin, die bis dato für die Ästhetik des Visuellen in ihrer Anwendung und Erscheinung im künstlerischen Artefakt zuständig gewesen war, nun von der Literaturwissenschaft, der Anthropologie, der Philosophie, den sogenannten Lebenswissenschaften, ja gar der Informatik marginalisiert wurde, wenn es um das Bild ging? Wurde die Kunstgeschichte etwa gerade auf Grund ihrer Zuständigkeit für das Ästhetische von Bild und Visualität marginalisiert? Schließlich produzieren weder die Kunstgeschichte noch die Ästhetik prognosefähiges Wissen für einen globalisierten Wissenschaftsbetrieb, im Gegensatz zu anderen Disziplinen, die sich dem Bild zugewandt oder über bildgebende Verfahren ihre Erkenntnis- wie Darstellungsstrategien verändert haben. Ich denke jedoch, daß diese Kompetenz oder Zuständigkeit für die Ästhetik noch keine ausreichende Erklärung bietet für die Randposition in Sachen Bildwissenschaft, in der sich die Kunstgeschichte wiederfindet.

Es soll nun nicht darum gehen, Argumente für eine zentrale Rolle der Kunstgeschichte in den bildwissenschaftlichen Clustern zu finden, und das nicht nur, weil ich durchaus nicht sicher bin, ob eine Position als Leitwissenschaft eines Clusterparadigmas von womöglich mäßig langer Halbwertzeit der Kunstgeschichte Vorteile strategischer Art bringen könnte. Mich interessieren vielmehr die Dynamiken und Hintergründe dieser Aufsplitterung der Zuständigkeiten für das Bild/das Visuelle zwischen der Disziplin Kunstgeschichte, dem

4 Vgl. W. J. T. Mitchell: The Pictorial Turn, in: Artforum 30, 1992, 89-94; dt.: Der Pictorial Turn, in: Christian Kravagna (Hrsg.), Privileg Blick. Kritik der visuellen Kultur, Berlin 1997, 15-40.

5 Gottfried Boehm: Die Wiederkehr der Bilder, in: ders. (Hrsg.), Was ist ein Bild?, München 1994, 11-38. 
Cluster Bildwissenschaft und der Indiscipline ${ }^{6}$ Visual Culture. Auch möchte ich versuchen, die Dichotomie von linguistic und pictorial oder visual turn respektive von Textualität/Sprache versus Visualität/Bild7 (wobei der Status dieser Begriffe nicht analog ist) aus dem Zentrum der Auseinandersetzung zu verschieben, denn ich nehme an, daß der Streit um das heuristische Primat von Sprache oder Bild seinerseits Symptom tiefergehender Konflikte und Veränderungen gegenwärtiger Wissenskultur ist.

\section{Essentialisierungen}

Die Visual Culture Studies haben in England und den USA bereits eine Diskursgeschichte akkumuliert, Reader und Einführungsliteratur produziert und dabei durchaus kein einheitliches Bild ihrer Theorien und Methoden hervorgebracht; eher noch könnte ihr erkenntnisleitendes Interesse auf einen Nenner gebracht werden. In einer extremen Verkürzung würde ich folgende Formulierung vorschlagen: In den Visual Culture Studies geht es um die Erforschung der begrenzten Ressource Sichtbarkeit. Die Implikationen sind vielfältig und verbunden mit einer politischen Agenda, die in der US-amerikanischen Variante eines Einwanderungslandes am deutlichsten hervortritt. Zur Ressource im politischen Sinne wurde Sichtbarkeit im Verbund mit den Identitätspolitiken, welche aus den Emanzipationsbewegungen der sechziger und siebziger Jahre hervorgegangen waren. Die Verschiebung vom Ringen um politische und ökonomische Teilhabe hin zur Sichtbarkeit ging vonstatten über die Idee, daß die Anerkennung marginalisierter Gruppen, in ihrer über die Differenzen von Geschlecht, Ethnie oder sexueller Orientierung determinierten ,Identität‘, mit einer Repräsentation innerhalb der jeweils hegemonialen Kultur oder auch gegen sie zu erkämpfen sei. Hier engagier(t)en sich die Cultural Studies. ${ }^{8}$ Aus den Cultural Studies entwickelten sich die Visual Studies oder Visual Culture Studies, welche diesen Aspekt der Repräsentation auf das Feld der Sichtbarkeit zuspitzten: „Such a history of visual culture would highlight those moments where the visual is contested, debated and transformed as a constantly challen-

6 W. J. T. Mitchell: Interdisciplinarity and Visual Culture, in: Art Bulletin 77, 1995, 540-544, hier 541 .

7 So geht es z. B. Sigrid Schade in ihrer Kritik am „Wunsch der Kunstgeschichte, Leitwissenschaft zu sein“, darum, die Geltung des semiologischen Ansatzes auch für die Analyse von Bildern zu verteidigen, mithin der Sprachwissenschaft das methodologische Primat zuzusprechen, vgl. Sigrid Schade: Vom Wunsch der Kunstgeschichte, Leitwissenschaft zu sein. Pirouetten im sogenannten „pictorial turn“, in: Zeitschrift des schweizerischen Instituts für Kunstwissenschaft - Zum 100jährigen Bestehen, Zürich 2001, 1-11.

8. Vgl. Stuart Hall (Hrsg.): Representation. Cultural Representations and Signifying Practices (1977), London/Thousand Oaks/New Delhi 1997. 
ging place of social interaction and definition in terms of class, gender, sexual and racialized identities. " Mirzoeff, der diese Bestimmung von Visual Culture geliefert hat, fügt ihr eine weitere hinzu, die zwei determinierende Elemente für die Notwendigkeit von Visual Culture Studies behauptet - die Neuen Medien und die Postmoderne: „Modern life takes place onscreen “10 beginnt seine Introduction to Visual Culture, und: „it is the visual crisis of culture that creates postmodernity, not its textuality." Postmoderne Kultur sei „most postmodern when it is visual". Mit der Repräsentation von Identitäten, den Neuen Medien, der mit ihnen einhergehenden visuell dominierten Alltagskultur und der Epochenbestimmung der Postmoderne ist der politische, mediale wie historische Rahmen der Visual Culture auf eine Weise abgesteckt, welche ihre Genese aus einer politischen Agenda der Identitätspolitiken der letzten Jahrzehnte verdeutlicht. In diesen Setzungen lauert jedoch bereits die Falle der Essentialisierungen: zum einen über die Implikationen der Identitätspolitiken (die auch bei den jeweils betroffenen Gruppen umstritten sind), zum anderen mit der behaupteten Dominanz des Visuellen, zuletzt auch mit der Behauptung einer essentiell postmodernen Visualität. Vehement hat sich Mieke Bal in ihrem Text Visual Essentialism and the Object of Visual Culture gegen diese Tendenz geäuBert. ${ }^{11}$ Mit „Visual Essentialism“, einer griffigen Formel, welche die Schlüsselproblematik der Debatten um die Politiken der Identität für ihre Kritik an den bisherigen Strategien und Definitionen von Visual Culture aufgreift, beschreibt sie den „purity-assuming cut between what is visual and what is not ${ }^{\text {"12 }}$; einen Schnitt, der auf vielen Ebenen der Versuche, den Gegenstand von Visual Culture zu definieren, zu beobachten ist und zwangsläufig nicht nur Ein- und Ausschlüsse zur Folge hat, sondern auch von dem Begehren angetrieben ist, eine unangreifbare Fundierung und Fixierung des Feldes zu erreichen. ${ }^{13}$ Bal greift in einem Moment in die interne Debatte der Visual Culture ein, als die Konsequenzen der Institutionalisierung dieser Indiscipline bereits beobachtbar sind: Der Druck, den Gegenstand zu definieren, die Kanonisierung von Objekten, Texten wie Methoden, das Patroullieren der Grenzen einer Disziplin, die keine ist, oder besser, keine werden sollte, führt ihr zufolge in eine falsche Richtung. Bal situiert den Gegenstand der Visual Culture Studies in jenem Beziehungsgeflecht selbst, das auch als Blickregime bezeichnet wird: Praktiken des Sehens, welche die Sehenden und das Gesehene nicht in einem Subjekt-Objekt-Ver-

9 Nicolas Mirzoeff: An Introduction to Visual Culture, London/New York 1999, 4.

10 Dieses und die beiden folgenden Zitate ebd., 3.

11 Mieke Bal: Visual Essentialism and the Object of Visual Culture, in: Journal of Visual Culture 1/2, 2003, 5-32.

12 Ebd., 6.

13 In ihrem Text analysiert sie diese definitorischen Strategien, die ich hier nicht im Detail verfolgen will, systematisch. 
hältnis gegenüberstellen, sondern in einer gegenseitigen Bedingtheit positionieren, die Effekt und Produktion in der Dynamik von Diskursen verschränkt. Worauf es mir nun ankommt, ist nicht die Auseinandersetzung darüber, mit welchem Instrumentarium die Analyse dieses, Gegenstandes' gelingen könnte, oder anders: Ich will nun nicht zurück in die Konfrontation von linguistic und visual turn, indem ich mit $\mathrm{Bal}$ argumentiere, die bekanntermaßen einen semiologischen, gemeinhin als textorientiert angesehenen Ansatz vertritt. ${ }^{14} \mathrm{Mir}$ kommt es hier auf ihre Kritik an Phänomenen an, die meines Erachtens in der Ausdifferenzierung der ,Familien'-Positionen von Kunstgeschichte, Bildwissenschaft und Visual Culture Studies eine zentrale Rolle spielen: Definition durch Abschottung in enger Wechselwirkung mit Identitätsbildung und Essentialisierung.

Die Bildwissenschaft des deutschsprachigen Raumes ist von anderen Begriffsbildungen geprägt. An der Formel des iconic turn, die Gottfried Boehm 1994, zwei Jahre nach der Erstveröffentlichung von Mitchells pictorial turn und ein Jahr nach Martin Jays Kritik ${ }^{15}$ einer Sehfeindlichkeit der französischen Philosophie, geprägt hat, wird bereits deutlich, wie sich die Perspektiven von jenen des visual turn unterscheiden. ${ }^{16}$ Mit dem Bild in der philosophischen Abstraktion des Ikonischen verschiebt sich buchstäblich der Blick von den Praktiken des Sehens oder dem Blickregime, zu einem Gegenstand, der nun auf den letztgültigen Nenner gebracht werden muß: „Was ist ein Bild?“"17 Die Motivationen für diese Frage nach einer Definition dessen, was ein Bild sei, sind vielfältig; verstanden aus dem Zusammenhang einer Entkräftung des linguistic turn jedoch geht es Boehm zufolge vordringlich um eine „neue(n) Verhältnisbestimmung, die das Bild nicht länger der Sprache unterwirft, vielmehr den Logos über seine eingeschränkte Verbalität hinaus, um die Potenz des Ikonischen“ zu erweitern und zu transformieren. ${ }^{18}$ In einem eleganten Sprachbild spricht er

14 So zum Beispiel in ihrem Buch: Reading Rembrandt. Beyond the Word-Image Opposition, New York 1991, mit dem sie den Methodenkanon der kunsthistorischen RembrandtForschung in Frage stellte.

15 W. J. T. Mitchell: The Pictorial Turn, in: Artforum, 1992, March, 89-94; Martin Jay: Downcast Eyes. The Denigration of Vision in Twentieth-Century French Thought, Berkeley/Los Angeles/London 1993.

16 Die, Vision' aus Jays Buchtitel wird im übrigen in der deutschen Rezeption in einer aufschlußreichen Verschiebung in das deutsche ,Bild' transformiert, von einer Relation, einem Akt oder einem Prozeß in einen Gegenstand; vgl. Horst Bredekamp: Drehmomente - Merkmale und Ansprüche des iconic turn, in: Christa Maar/Hubert Burda (Hrsg.), Iconic Turn. Die neue Macht der Bilder, Köln 2004, 15-26, hier 16.

17 Vgl. den von Gottfried Boehm herausgegebenen Band: Was ist ein Bild?, München 1994.

18 Gottfried Boehm: Jenseits der Sprache? Anmerkungen zur Logik der Bilder, in: Maar/ Burda 2004 (wie Anm. 16), 28-43, hier 30. Boehm argumentiert subtil mit den Argumenten der Sprachkritik, u. a. mit der Sprachphilosophie Wittgensteins, um dieses Verhältnis aus dem Ungenügen der Sprache, Sinn auch jenseits von Eindeutigkeiten zu generieren, als ein Notwendiges zu begründen. 
vom „Schatten der Sprache [...], der sich über das Ikonische legt." ${ }^{19}$ Mitchell hatte eine andere Metapher für dieses Verhältnis gefunden, die nicht nur eine andere Gewichtung von Sprache und Bild aufweist, sondern auch von der Figur des Ikonischen als Opfer des Sprachlichen weg hin zu den Kommunikationswegen zwischen beiden, vom Kategorialen hin zur Praxis einer Beziehung führt: „Einem Bild einen Ausdruck zu verleihen, bedeutet das kunstvolle Legen bestimmter Spuren, die es uns gestatten, als Bauchredner aufzutreten und dem Bild eine Beredtheit, und zwar vor allem eine nichtvisuelle, sprachliche Beredtheit zu verleihen. "20 Dagegen liest sich Beltings Versuch der kategorialen Festlegung eines Primats des Bildes wie ein verzweifelter Aufruf zur Rettung des Körpers gegen seine medientechnologische Virtualisierung: „Schon muß man darum kämpfen, den Beweis führen zu können, daß der Bildbegriff allein als anthropologischer Begriff gerechtfertigt war. Nur hier, und nicht im technologischen Diskurs, läßt sich der Doppelsinn des Bildes - des Bildes an der Wand und des Bildes im Kopf, die dennoch beide interagieren - begründen. Wir selbst sind als lebende Medien der ,Ort der Bilder" und nicht die Apparate. "21 Mit rhetorischer Vehemenz treibt Belting nicht erst seit seiner Bildanthropologie von 2001, die Hanne Loreck zu den „antisemiologischen und antidekonstruktivistischen Rettungsversuche(n) eines emphatischen Bild- wie Körperbegriffs“ zählt ${ }^{22}$, die „Bild“- oder „Bilderfrage“ voran. Er beklagt nicht nur, wie Boehm, eine Verarmung durch allzu großes Vertrauen in die Logik der Sprache, sondern weist auf ein ganzes Korrelat von Feinden, die entweder Körperbild oder Bildkörper rauben: die „digitalen Bildwelten“ virtueller Körper, der „genetische Code“, der uns „in einem analogen Ikonoklasmus die visuelle Evidenz dessen, was wir heute vom Körper wissen“, entzieht. ${ }^{23}$ Der Modus einer Formulierung wie der ,Bilderfrage', die im übrigen häufiger im bildwissenschaftlichen Diskurs auftaucht, läßt strukturell an andere ,Fragen' denken, in denen es um Ängste, Faszinosa, Rätsel, um eine Wiederkehr des Verdrängten geht: die Frauenfrage, die Arbeiterfrage, die Judenfrage. Fragen dieser Art rufen nach kategorialen Antworten, nach Definitionen. Sie heben ab auf ein

19 Ebd., 33.

20 W. J. T. Mitchell: Was ist ein Bild?, in: Volker Bohn (Hrsg.), Bildlichkeit, Frankfurt a. M. 1990, 17-68, hier: $52 \mathrm{f}$.

21 Hans Belting: Echte Bilder und falsche Körper - Irrtümer über die Zukunft des Menschen, in: Maar/Burda 2004 (wie Anm. 16), 350-364, hier 363.

22 Vgl. Hans Belting, Bild-Anthropologie. Entwürfe für eine Bildwissenschaft, München 2001. Vgl. dazu die ,differenztheoretische Lektüre' von Hanne Loreck: Bild-Andropologie? Kritik einer Theorie des Visuellen, in: Susanne von Falkenhausen/Silke Förschler/Ingeborg Reichle/Bettina Uppenkamp (Hrsg.), Medien der Kunst. Geschlecht, Metapher, Code. Beiträge der 7. Kunsthistorikerinnen-Tagung in Berlin 2002, Marburg 2004, 12-26, hier 17.

23 Belting 2004 (wie Anm. 21), 351. 
jeweils Anderes, das es ein- wie auszugrenzen und zu kontrollieren gilt. Sie tun das, was Bal Essentialisierung nennt: Sie schotten ab, suchen nach Reinheit des als erklärungsbedürftig ausgewiesenen Phänomens im Sinne des Unvermischten und: Sie machen Politik. Hanne Loreck begründet triftig, daß es in der Bildwissenschaft beim Rekurs auf ein Humanum als anthropologische Invarianz um die Abwehr der kritischen Potentiale jener Differenztheorien geht, die sich der Kritik an totalisierenden Systemen und Diskursen gewidmet haben. Die Rückkehr zu Universalien mit deutlich prämodernen Zügen wird da jedoch kaum Heilung bringen, auch wenn diese in postindustriellen und posthistorischen Gewändern auftreten, wie dies gerade auch in der Mythenbildung zu digitalen Technologien und ihren naturwissenschaftlichen Anwendungen besonders in den bildgebenden Verfahren der Fall ist, welche dieselbe Perspektive zur Legitimation aufzurufen in der Lage sind. Symptomatisch wäre hier z. B. der Mythos der Präsenz: Appliziert auf die technischen Möglichkeiten der Realzeit-Repräsentation - Real-Time als radikale Mimesis im Temporalen werden die neuen Kommunikationstechnologien über die Mythisierung dieser bildtechnologisch realisierten Unmittelbarkeit und Gegenwärtigkeit naturalisiert und verschaltet mit dem Invarianz-Apriori der Anthropologie. Derart kann, über einen ontologisierten Bildbegriff, auch die Entwicklung von Techniken der Kontrolle und Steuerung (Psycho- wie Kulturtechniken), zwischen Neurowissenschaft, Bioengineering, Genetik, Kybernetik und Social Engineering als Aufklärung und Bewahrung des Humanum verkauft werden.

So zieht sich das Bild als heuristisches Krisensymptom zwischen Paradigma und Metapher in den letzten zehn Jahren durch Human- wie Naturwissenschaften. Ist es zur Projektionsfläche für einen Traum von Ganzheit geworden, quer zu den verwirrenden Bedingungen wissenschaftlicher Positionierungen im Zeitalter der „Exzellenz “24 und zu Utopien sozialer Wirksamkeit im globalen Postindustrialismus? Geht es bei der diskursiven Omnipräsenz des Bildes in den Wissenschaften als einer Art Letztinstanz dessen, was der Aufklärung bedarf und dann umschlägt in die Naturalisierung eines Nichthinterfragbaren,

24 „Excellence is non-referential, a unit of value entirely internal to the system, [...] All the system requires is for activity to take place, and the empty notion of excellence refers to nothing other than the optimal input/output ratio in matters of information." Bill Readings: Be Excellent. Culture, the State, and the Posthistorical University, in: Alphabet City 3, 1993, zit. nach: Stephen Melville: Art History, Visual Culture, and the University, in: October 77, 1996, 52-54, hier 52 (Melvilles Statement zum umstrittenen Visual Culture Questionnaire). Melville fragt sich im übrigen, ob Visual Culture Studies eher als letzter Widerstand der Universitätder Kultur gegen die Universität der Exzellenz zu verstehen sei oder als Appropriation dessen, was von der Universität der Kultur noch übriggeblieben sei, durch die Universität der Exzellenz. Zehn Jahre später ist noch nicht klar, ob die deutsche Universität der Exzellenz sich überhaupt mit Kultur beschäftigen wollen wird. Zur ,University of Excellence' vgl. auch Bill Readings: The University in Ruins, Cambridge 1996. 
um ein Befragen wie Bestätigen und Beschwören eines Phänomens, das Brüche und Widersprüche in heutigen intellektuellen Konstruktionen zu überblenden vermag?

\section{Und die Kunstgeschichte?}

Vereinfacht könnte man sagen, daß die Kunstgeschichte ein Kind jener Epoche sei, in welcher die klassifizierende Beobachtung zum Leitmotiv von Wissenschaften wie der Botanik geworden war. Die Klassifizierungskategorien sind bekannt: Epochen, Gattungen, Stile, Regionen, Händescheidung. Aufbrüche aus der Enge dieser Erkenntnisziele, die in erster Linie der einsetzenden Sammlungs- und Museumsdynamik geschuldet waren (bzw. umgekehrt) gab es seither viele; sie haben das Fach bereits seit dem späten 19. Jahrhundert differenziert und anderen, darunter vor allem kulturhistorischen Horizonten angenähert.

Aus europäischer Perspektive kann es deshalb jene allzu eindimensionale Polarisierung zwischen Visual Culture und Kunstgeschichte kaum geben, welche in den Debatten um Visual Culture versus Kunstgeschichte im angloamerikanischen Raum zur Profilierung der Visual Culture als antielitär, multikulturell, postkolonial und demokratisch gegenüber der Negativfolie einer ästhetisch, elitär, formalistisch bis hegelianisch, künstlergenialisch und nationalistisch orientierten Kunstgeschichte eingesetzt wurde. ${ }^{25}$ Selbstverständlich gab und gibt es alle diese „Kunstgeschichten“, oder besser diese Narrative in der Kunstgeschichte, ebenso wie jene anderen, die wiederum dem ursprünglich politisch-kritischen Programm der Visual Culture Studies nahe stehen. Daraus läßt sich jedenfalls ein Schluß ziehen: Die Disziplin oder In/disziplin ist es nicht, die bereits den als fortschrittlich erachteten Erkenntnishorizont determiniert. Für die Kunst wäre im übrigen eine analoge Formulierung die, daß weder ein bestimmter Formenkanon (z. B. die Montage, Peter Bürgers Kriterium der Avantgarde ${ }^{26}$ ) noch bestimmte Medien (z. B. der Film für Walter Benjamin ${ }^{27}$ ) Garanten für das politisch ,richtige' Programm sein können. Um-

25 Hier kann als symptomatisches Beispiel für den daraus resultierenden Legitimationsdruck der amerikanischen Kunstgeschichte die Methodendiskussion angeführt werden, die in einigen Nummern des Art Bulletin in den Jahrgängen 1994, 1995 und 1996 stattfand. Unter Titeln wie A Range of Critical Perspectives (Art Bulletin, Dezember 1995) oder Aesthetics, Ethnicity, and the History of Art (ebd., Dezember 1996) diskutierten Vertreter der Kunstgeschichte und der Visual Culture Studies.

26 Vgl. Peter Bürger: Theorie der Avantgarde, Frankfurt a. M. 1974, bes. 98. Vgl. dazu auch: W. Martin Lüdtke (Hrsg.): „Theorie der Avantgarde“ Antworten auf Peter Bürgers Bestimmung von Kunst und bürgerlicher Gesellschaft, Frankfurt a. M. 1976.

27 Womit einmal mehr auf Walter Benjamins Kunstwerk-Aufsatz angespielt sei: Das Kunstwerk im Zeitalter seiner technischen Reproduzierbarkeit (1936), dt. Erstveröffentlichung Frankfurt a. M. 1955. 
gekehrt gilt jedoch die banale Weisheit, daß der Erkenntnishorizont die Methode(n) organisiert, ob ausgewiesenermaßen oder nicht.

Eine anthropologisch verstandene Kunstgeschichte würde Kunst als Grundbedürfnis des Menschen erforschen: der Mensch und sein Kunst-Bedürfnis als kategoriale Invarianzen, in merkwürdiger Unschlüssigkeit kombiniert mit dem historischen Wandel der derart bestimmten Kunst. Kunst würde in dieser Perspektive bezeugt bis zu den frühesten kulturellen Artefakten und Bildzeugnissen, in einer ahistorischen Entgrenzung der Kategorie Kunst. Eine solche Bewegung jedoch würde die Fähigkeit der Kunstgeschichte zur Reflexion ihrer Gegenstandsbestimmung, wie sie mit der Historisierung der Kategorie Kunst selbst gegeben ist, aushöhlen und in der Konsequenz ihr kritisches Potential, das auch daraus erwächst, den sich wandelnden Status von Kunst als soziokulturelle Praxis zu befragen. Sie wiche zudem weit hinter jene Position zurück, die seit dreißig Jahren bis in die Einführungen in das Fach vorgedrungen ist und die Martin Warnke nüchtern auf einen Nenner gebracht hat: „,Kunst“ aber ist ein abstrakter Begriff. Thre konkrete Erscheinungsform ist das Kunstwerk, ein Artefakt, das sich von anderen menschlichen Artefakten dadurch unterscheidet, daß ihm die besondere Eigenschaft, Kunst zu sein, zugesprochen wird. “28 Heute sind auch die Prozesse dieser Zuschreibung Gegenstand der Kunstgeschichte. Eine derartige relational orientierte Bestimmung hat noch einen weiteren Vorteil: Sie verabsolutiert weder den Sehsinn noch die Visualität und auch nicht das Bild und erspart sich so jene grundlegenden Probleme von Visual Culture Studies und Bildwissenschaft, die vom Definitionsimpetus aus der Abgrenzung gegenüber der Sprache herrühren.

Nach den Positionierungsmanövern der Familienmitglieder nun zu den möglichen - Beziehungen: Berührungen zwischen Kunstgeschichte und Bildwissenschaft ergäben sich dort, wo die Kunstgeschichte die strenge Historizität ihres Gegenstandes in der eben skizzierten Reflexionsdynamik für eine Bildgeschichte ins Spiel brächte, ohne auf eine Bildgeschichte beschränkt zu sein. Kunstgeschichte brächte so die historische Dimension als kritische ins Spiel, nicht jedoch als Ergänzungsfigur zur ontologisierenden Invarianz anthropologischer Prägung. Auf der Ebene eines diskurshistorisch orientierten Konstruktivismus können sich hingegen Kunstgeschichte und Visual Culture Studies wechselseitig erhellen: Kunstgeschichte stellt die Kunst in die Diskursformationen des Visuellen und ihre Geschichte ein und reicht insofern nicht nur über die Kunst, sondern auch über ein allgemein Visuelles hinaus. In der Kontextforschung, deren Fragehorizonte durchaus Gemeinsamkeiten mit den Visual Culture Studies aufweisen, ist dies bereits etablierte kunsthistorische Praxis.

28 Martin Warnke: Gegenstandsbereiche der Kunstgeschichte, in: Hans Belting/Heinrich Dilly/Wolfgang Kemp/Willibald Sauerländer/Martin Warnke (Hrsg.), Kunstgeschichte. Eine Einführung, Berlin ${ }^{3} 1988,19-44$, hier 24 [Hervorhebung SvF]. 
Umgekehrt jedoch führt auch eine denkbare Bewegung von der Kunstgeschichte zurück zu den Visual Culture Studies: Gerade der Status von Kunst als einer ausgewiesenen Praxis mit dem entsprechenden Apparat (von der Ausbildung bis zu den Institutionen), also als diskursives wie außerdiskursives Faktum qua synchroner wie diachroner Zuschreibung, vermag wie in einem Brennglas exemplarische Aufschlüsse für den zentralen Gegenstand der Visual Culture Studies zu bündeln: über die Praktiken des Sehens im Felde der Macht. 